\title{
eJRIEPS
}

Ejournal de la recherche sur l'intervention en éducation physique et sport

$37 \mid 2016$

Varia

\section{L'entraînement et l'enseignement de la prise de décision tactique dans les sports collectifs}

L'usage des situations de jeux réduits à l'épreuve de modèles théoriques et de résultats de la recherche en sciences du sport

\section{Gilles Kermarrec et Cédric Roure}

\section{(2) OpenEdition}

1 Journals

Édition électronique

URL : https://journals.openedition.org/ejrieps/1154

DOI : 10.4000 /ejrieps. 1154

ISSN : 2105-0821

Éditeur

ELLIADD

Édition imprimée

Date de publication : 1 janvier 2016

Référence électronique

Gilles Kermarrec et Cédric Roure, «L'entraînement et l'enseignement de la prise de décision tactique dans les sports collectifs », eJRIEPS [En ligne], 37 | 2016, mis en ligne le 01 janvier 2016, consulté le 08 décembre 2022. URL : http://journals.openedition.org/ejrieps/1154; DOI : https://doi.org/10.4000/ ejrieps. 1154

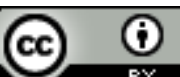

Creative Commons - Attribution 4.0 International - CC BY 4.0 https://creativecommons.org/licenses/by/4.0/ 
eJRIEPS 37 janvier 2016

\title{
L'entraînement et l'enseignement de la prise de décision tactique dans les sports collectifs
}

L'usage des situations de jeux réduits à l'épreuve de modèles théoriques et de résultats de la recherche en sciences du sport

\author{
Gilles Kermarrec ${ }^{*} \&$ Cédric Roure ${ }^{* *}$ \\ *Centre de Recherche en Education Apprentissages et Didactique (EA 3875-CREAD) \\ Université Européenne de Bretagne, France \\ ** Groupe Interdisciplinaire de Recherche sur la Socialisation, l'Education et la Formation \\ (GIRSEF), Université Catholique de Louvain, Belgique.
}

\section{Résumé}

L'objectif de cet article est d'interroger l'usage de situations de jeux réduits (SJR) pour la formation tactique en sports collectifs, à l'aune de modèles pédagogiques et de modèles théoriques de la prise de décision qui peuvent leur être associés. Trois critères sont mobilisés : les fondements théoriques des modèles convoqués, les acquisitions visées et les mécanismes d'apprentissage valorisés, leurs intérêts et limites. L'analyse comparative porte initialement sur le modèle de la pédagogie de la compréhension, valorisant l'apprentissage explicite, et sur le modèle de la pédagogie par manipulation des contraintes de l'environnement, valorisant l'apprentissage implicite. Puis, le modèle de la prise de décision intuitive est convoqué car il met en avant la complémentarité des feedbacks explicites et implicites. La synthèse de la littérature met en évidence que l'utilisation de SJR s'appuie sur des présupposés théoriques parfois contradictoires. Pour autant, apprendre à identifier des configurations de jeu articulant des aspects moteurs, perceptifs, cognitifs et émotionnel semble devenir une visée commune. Enfin, le rôle tenu par les intervenants dans ces situations se différencie selon les mécanismes d'apprentissage sollicités, qui peuvent être implicites ou explicites.

Mots-clés : enseignement des sports collectifs, jeux réduits, prise de décision, habiletés tactiques, apprentissage. 


\section{eJRIEPS 37 janvier 2016}

\section{Introduction}

Récemment de nombreuses publications dans la littérature en sciences du sport ont mis en avant l'intérêt des jeux réduits pour la formation à la décision tactique des joueurs en sports collectifs (e.g., David, Araujo, Correia, \& Vilar, 2013). Cet intérêt pour une forme de pratique connue de longue date constituerait-il un effet de mode? Dans quelle mesure les jeux réduit favorisent-ils le développement des capacités de décision tactique? Les capacités de décision tactique renvoient à des choix en action, et se distinguent des capacités de décision stratégique qui se manifestent lors de décisions prises en amont de l'action (Gréhaigne, Godbout, \& Bouthier, 2001 ; Raab, 2007). Les capacités de décision tactique nécessaires à la performance dans les sports collectifs seraient développées par des situations désignées par différents vocables tels que jeux réduits, situations jouées, situations authentiques, situations complexes, situations de résolution de problème, que nous proposons de réunir par l'appellation de situations de jeux réduits (SJR). Les SJR en sports collectifs désignent les situations dont l'enjeu fondamental réside dans la coordination d'actions au sein d'un rapport d'opposition dans le but de récupérer, conserver, faire progresser le ballon vers la zone de marque et de marquer (Gréhaigne \& al., 2001). Quelque soit l'effectif utilisé (du 2 contre 2 au 11 contre 11, cf. Little \& Williams, 2006), les SJR se caractérisent toujours par une cible à atteindre avec un ballon dans le camp adverse et une cible à défendre dans son propre camp, une surface de jeu délimitée, des partenaires et des adversaires en nombre équivalent ou non, un temps de jeu, un règlement et des contraintes qui définissent des rôles, et enfin un système de score ou de décompte des points (David \& al., 2013).

$\mathrm{Au}$ delà du fait que ces situations soient largement diffusées dans les pratiques de formation, le but de cette revue de question est de tenter d'expliquer pourquoi les jeux réduits, comme pratiques d'intervention, sont actuellement plébiscités par des auteurs mobilisant différents modèles scientifiques relatifs au développement de la prise de décision. Pour ce faire, nous avons examiné la littérature scientifique récente (à partir des années 2000) en sciences du sport, en croisant des mots-clés (enseignement des sports collectifs, jeux réduits, prise de décision, habiletés tactique). Au sein du corpus de travaux obtenu, nous avons identifié et regroupé des contributions qui, en dépit d'objets d'étude proches ou similaires, se fondaient sur trois grandes orientations théoriques. Ainsi, l'usage des SJR a initialement été étudié dans le cadre d'une pédagogie de la compréhension (souvent associée au modèle Teaching Games for Understanding) valorisant la construction explicite de connaissances par une alternance entre action et 


\section{eJRIEPS 37 janvier 2016}

verbalisation sur l'action (Gréhaigne, Richard, \& Griffin, 2005). Dans le cadre d'une approche pédagogique fondée sur la manipulation des contraintes de l'environnement (Constraints-Led Approach, cf. David, et al., 2013), le développement des capacités tactiques consisterait en une adaptation comportementale implicite, sans recours à une boucle cognitive (Araujo, Travassos \& Vilar, 2010). Enfin, des travaux s'inscrivant dans le paradigme de la prise de décision intuitive (Raab, 2007) ont apporté leur contribution à l'identification des mécanismes et des acquisitions susceptibles d'être développées par ces SJR, et ont proposé une perspective syncrétique en mettant en avant l'alternance et la complémentarité de l'apprentissage explicite et implicite.

L'analyse, puis la synthèse de ces travaux, a été systématiquement organisée en trois temps : a) les modèles et les construits sous-tendant l'usage des jeux réduits en sports collectifs ; b) le(s) processus d'apprentissage favorisé(s) et les acquisitions visées ; c) l'intérêt et les limites pour la formation tactique en sports collectifs.

\section{Les situations de jeux réduits et la construction de connaissances par la compréhension}

L'utilisation des SJR en sports collectifs s'inscrit d'une part dans la tradition francophone d'une approche tactique de la formation, d'autre part dans la continuité d'un modèle pédagogique né aux Etats-Unis (pour une revue détaillée et récente des origines et des avancées des recherches relatives à l'approche tactique en sport collectif, voir Gréhaigne \& Nadeau, 2015).

\subsection{Fondements théoriques}

En France, l'approche tactique de la formation en sports collectifs est notamment fondée sur une analyse des jeux collectifs, initiée par les travaux de Deleplace (1979), de Mérand (1989) ou de Gréhaigne (1989). Avec les travaux de Bouthier (1986) conduisant à proposer une Pédagogie des Modèles de la Décision Tactique (PMDT), ces précurseurs ont engagé un renouvellement de la pédagogie de l'enseignement des sports collectifs. Diffusée notamment dans le contexte scolaire de l'Education Physique, cette pédagogie valorise la compréhension du jeu (le jeu de l'équipe adverse et celui de sa propre équipe) et «la recherche de principes et de règles d'action » par l'élève (Gréhaigne \& Roche, 1990, p.68).

Aux Etats-Unis, des propositions pédagogiques alternatives à une approche traditionnelle privilégiant l'apprentissage des techniques comme point de départ de l'enseignement des sports collectifs ont abouti au modèle Teaching Games for Understanding (TGfU). La 


\section{eJRIEPS 37 janvier 2016}

proposition de Bunker \& Thorpe (1982) est considérée comme fondatrice de cette approche (Kirk \& Mc Phail, 2002 ; Raab, 2007), ou pour le moins un moment clé dans la diffusion du modèle (Butler \& Ovens, 2015). Dans ce modèle, une situation de jeu constitue le point de départ et le point d'arrivée d'une séquence de formation. Elle permet d'identifier clairement les problèmes rencontrés et donne aux connaissances apportées par le formateur le statut d'une réponse à un besoin clairement identifié. Cette approche « centrée sur l'apprenant » considère qu'un élève peut pratiquer efficacement un jeu collectif même à partir d'un registre technique assez limité (Thorpe, 1990).

Dans la continuité de ces travaux francophones et anglophones, un des usages des SJR dans le cadre d'une pédagogie de la compréhension se caractérise par une structuration de toute séquence de formation qui (a) commence par des situations authentiques de jeu (réduit ou non), (b) se nourrit d'observations du jeu et de feedbacks explicites, (c) se poursuit par des moments de verbalisations de type « débat d'idées », aboutissant à la formulation d'un projet d'action, (d) se termine par un retour au jeu afin que les élèves valident où invalident le projet d'action et les règles d'actions issus des verbalisations.

\section{2. Processus d'apprentissage et acquisitions}

Le processus d'apprentissage peut être qualifié d'explicite (Raab, 2007), car les moments de verbalisations à propos du jeu sont considérés comme essentiels dans le processus d'apprentissage. Ces débats d'idées (e.g., Zghibi, Guinoubi, Bennour, \& Moheiddine, 2013) sont dirigés par un formateur encourageant descriptions et analyses, ou organisés entre pratiquants attestant d'expériences différentes dans le jeu sportif enseigné (e.g., Darnis-Paraboshi, Lafont \& Menaut, 2006). En considérant que le développement de la prise de décision est favorisé à chaque fois que la réflexion sur l'action est demandée, Gréhaigne \& Wallian (2007, p. 16) défendent l'utilisation des SJR comme un lieu de construction de connaissances.

Ces SJR proposées peuvent être très proches de la situation de performance (le match), ou très structurées, ciblées sur une forme de jeu identifiée comme solution au problème posé (Nachon \& Musard, 2009). L'usage préconisé ici des SJR permettrait de donner du sens aux connaissances apportées par le formateur ou découvertes par les pratiquants, de valider ou d'invalider des connaissances antérieures, d'améliorer leur organisation des connaissances en identifiant des hiérarchies dans les choix, voire de construire des connaissances généralisables en sports collectifs (Mitchell \& Oslin, 1999).

Pour évaluer les effets de cet usage des SJR, les études réalisées, notamment en contexte scolaire, utilisent des tests moteurs et verbaux et des observations. Elles 


\section{eJRIEPS 37 janvier 2016}

mettent en évidence une augmentation des connaissances et de la complexité des projets d'action sous l'effet du dispositif, avec des variations en fonction du niveau de classe ou d'expertise (Nachon \& Musard, 2009), ou du niveau de développement cognitif (Darnis, \& al., 2006). Quand il s'agit de mesurer les effets de la pédagogie de la compréhension sur l'efficacité réelle des actions individuelles et collectives, les résultats semblent plus nuancés: par exemple dans une étude longitudinale réalisée lors de 8 séances de football, les joueurs devenaient effectivement plus efficaces dans les trois dernières séances, comme s'ils ajustaient progressivement leurs projets d'action verbalisés en dehors de l'action, à leurs capacités réelles en action (Zghibi, \& al., 2013).

2. 3. Intérêts et limites

Dans leur revue de la littérature, Chow, Davids, Button, Shuttleworth, Renshaw, \& Araújo (2007), ainsi que Gréhaigne \& Nadeau (2015), pointent la difficulté à mettre clairement en évidence l'efficacité du modèle TGfU pour la formation tactique en sports collectifs. Des études récentes montrent les décalages entre les objectifs de formation déclarés et les pratiques réelles (e.g., Poussin, Dénervaud, \& Lenzen, 2014). De plus, l'utilisation des SJR dans le cadre d'une pédagogie de la compréhension peut être interrogée à l'aune du statut des connaissances et du modèle de la prise de décision sous-jacent. En effet en considérant les connaissances comme les composants essentiels d'une prise de décision efficace, cette approche pédagogique ne renverrait-elle pas à une conception cognitiviste de la prise de décision, où l'action serait subordonnée à la cognition (voir le modèle de l'acte tactique revisité par Gréhaigne, Marle \& Zérai, 2014) ?

Les limites du modèle cognitiviste de la prise de décision ont déjà été pointées (pour des revues en langue française, voir Bossard \& Kermarrec, 2010 ; Macquet \& Fleurance, 2006) :

les capacités tactiques ne se réduisent pas à des capacités verbales (Araujo, Travassos \& Vilar, 2010), ce qui peut expliquer des décalages entre projet d'action et efficacité de l'action ;

- l'apprentissage explicite serait peu compatible avec les contraintes de pression temporelle (Masters, 2000), car la mobilisation des connaissances lors des SJR risquerait d'interrompre ou de perturber le couplage perception-action ;

des conditions d'apprentissage explicites conduiraient à une amélioration de la pertinence des choix, mais pas à une amélioration de leur rapidité (Farrow \& Fournier, 2005 ; Raab, 2003) ; 


\section{eJRIEPS 37 janvier 2016}

- le fonctionnement cognitif du joueur ne serait pas rationnel, en exécutant un plan d'action préalablement défini, mais emprunt de singularité et de subjectivité (Mouchet et Bouthier, 2006), car le joueur vit les situations de jeu de son propre point de vue (histoire personnelle, techniques préférentielles, fatigue ressentie), et la décision tactique serait intuitive (Mouchet, 2014).

Les tenants d'une approche tactique de la formation en sport collectifs ont déjà répondu à ces critiques en soulignant l'importance de cycles de pratique longs, de 6 à 8 séances minimum (Turner, 1996 ; Gréhaigne, Richard \& Griffin, 2005 ; Gréhaigne \& Wallian, 2007). Dans le cadre des SJR, pour accompagner ce développement, l'entraîneur ou l'enseignant peut aider les élèves à prendre des repères dans l'environnement. Caty, Meunier et Gréhaigne (2007) font l'hypothèse qu'une aide au repérage des configurations de jeu favoriserait la construction d'une image opérative. Ils font référence aux travaux d'Ochanine (1975, cité par Caty et al.) décrivant l'image opérative comme une image partielle, économique, mais suffisante de la situation, articulant des repères perceptifs, cognitifs et moteurs.

Ces évolutions les plus récentes prônant un apprentissage du repérage de configurations de jeu, sont en adéquation avec une perspective constructiviste (voir pour une analyse approfondie Griffin \& Butler, 2005 ; Singleton, 2009), voire « sémio-constructiviste " (Gréhaigne \& Nadeau, 2015), car :

le pratiquant acquiert des modalités de réponses complexes formulées sous formes d'heuristiques (Gréhaigne, Billard \& Laroche, 1999), et non des connaissances transmises comme des référentiels décisionnels exhaustifs;

- la connaissance est une construction de significations personnelles, originales et singulières (Kirk \& Mc Phail, 2002 ; Nachon \& Musard, 2009), et non une norme ou une procédure imposée ;

les connaissances et les décisions sont flexibles, issues d'une intelligence pratique, et connectées aux pouvoirs moteurs disponibles (Gréhaigne \& Wallian, 2007) ;

- la construction de connaissances est plus un moyen qu'une fin, en favorisant la perception et la différenciation des configurations prototypiques (Gréhaigne, Caty \& Gobout, 2010).

Gréhaigne, \& al. $(2010,2015)$ pointent le passage d'un enseignement pour la compréhension (Teaching Games for Understanding), à un apprentissage par la compréhension («Learning Games through Understanding»). Cette évolution se situe « en droite ligne avec une perspective cognitiviste et constructiviste » (Gréhaigne \& 


\section{eJRIEPS 37 janvier 2016}

Nadeau, 2015, p. 131), cognitiviste en raison du statut des connaissances, ressources pour l'action, constructiviste en raison du rôle actif de l'apprenant dans l'identification des problèmes posés par le jeu.

\section{Les situations de jeux réduits et l'adaptation aux contraintes de l'environnement}

L'analyse précédente conduit à questionner l'usage des SJR, en pointant le débat relatif à la part de la pratique et de la réflexion sur la pratique pour la formation tactique. A l'inverse, des travaux qui s'inscrivent dans une perspective écologique de la prise de décision valorisent l'adaptation par la pratique sous l'effet des contraintes imposées par les SJR. Cette approche de l'enseignement des sports collectifs basée sur la manipulation des contraintes de l'environnement (Constraints-Led Approach, Davids, Araújo, Correia, \& Vilar, 2013) valorise l'importance de la conception ou du design des SJR en amont de la pratique par le formateur.

3. 1. Fondements théoriques

L'usage des SJR dans le cadre d'un pédagogie par adaptation aux contraintes de l'environnement (pour une revue détaillée, voir Davids \& al., 2013) est sous-tendu par le modèle écologique de la prise de décision (Araujo, Davids, \& Hristovski, 2006). Initialement mobilisé dans la recherche en sport par Araujo, Davids \& Serpa (2005), ce modèle définit la prise de décision comme un couplage entre les ressources mobilisées par l'individu et l'environnement avec lequel il interagit. La prise de décision reposerait sur l'identification d'affordances (Brunswik, 1956), c'est à dire des informations utiles pour réduire l'incertitude issue des multiples combinaisons entre les contraintes externes et les ressources internes du pratiquant. Aussi, la dynamique du couplage dans un même environnement se traduit par une stabilisation progressive des informations utilisées et des réponses comportementales (pour une description détaillée du modèle de Brunswick, voir Araujo \& al., 2005). Dans les SJR, la variété des contraintes imposées au pratiquant dans l'environnement (distances, nombre de cibles, sources de ballon, ...) constituent autant d'affordances potentielles.

\section{2. Processus d'apprentissage et acquisitions}

Le processus d'apprentissage procède d'une adaptation d'un sujet à son environnement grâce à l'identification d'affordances, et qui se traduit par l'émergence d'invariants comportementaux au cours des expériences. Ces invariants consistent en des réponses spécifiques, contextualisées et non en des connaissances relativement générales et explicitées (Araujo, \& al., 2005). L'apprentissage de ces invariants est décrit comme un 


\section{eJRIEPS 37 janvier 2016}

processus implicite reposant sur l'exploration des opportunités d'action offertes par les contraintes de l'environnement, sans recours à une boucle cognitive couteuse en temps et en attention.

Les effets de la pratique des SJR ont été étudiés dans le cadre de travaux identifiés sous l'acronyme SSCG (Small-Sided Conditioned Games). Les résultats de l'apprentissage en SJR ont été décrits à partir de méthodes de capture et d'analyse de mouvements sur vidéo et à partir d'indicateurs comportementaux individuels ou collectifs (Travassos, Davids, Araujo, \& Esteves, 2013). Cependant, certains chercheurs (e.g., Folgado \& al., 2014) ont considéré que des indicateurs individuels (e.g., les déplacements de chaque joueur) étaient réducteurs, et que les aspects tactiques de la performance d'une équipe devaient être étudiés de façon holistique, à l'aide d'indicateurs collectifs tels que l'espace de jeu occupé par une équipe, le nombre de passes effectuées, le nombre de joueurs en interaction, ou les circuits préférentiels du ballon. Ainsi, dans plusieurs études, les positions de chacun des joueurs d'une équipe au cours d'une SJR ont été systématiquement recueillies, avec en général une fréquence de $25 \mathrm{hz}$. Ces positions permettent de représenter l'espace de jeu effectif sous la forme d'un polygone, et on peut calculer le centroïde de ce polygone. Le centroïde peut être un bon indicateur du mouvement d'une équipe, et des mouvements au sein de l'équipe, car il représente un point théorique, déterminé à partir de la moyenne arithmétique de tous les points (les différents joueurs) constituant le polygone (l'équipe). Enfin, un indice d'élasticité (ou Stretch Index) a été utilisé pour caractériser le comportement d'une équipe : il est calculé en additionnant, à chaque moment du jeu, la longueur de l'équipe (la distance entre les deux joueurs les plus éloignés d'une même équipe, sur un axe dans le sens de la profondeur du terrain) et la largeur de l'équipe (la distance entre les deux joueurs les plus éloignés d'une même équipe, sur un axe dans le sens de la largeur du terrain) d'une équipe (Folgado et al., 2014).

Les résultats de ces études (pour une revue voir Davids \& al., 2013) ont mis en avant des relations entre les contraintes manipulées dans les SJR et des invariants comportementaux. Par exemple, la manipulation des droits des joueurs en phase offensive (jeu libre vs jeu en deux touches de balle vs jeu en quatre touches de balle) influence le nombre de passes réalisées et le nombre de joueurs impliqués dans une même action (Ameida, Feirreira, et Volossovitch, 2013). Dans un SJR (5 contre 5), l'augmentation du nombre de cibles ( $1 \mathrm{C}$ vs $3 \mathrm{C}$ ) augmente la distance entre les centroïdes des deux équipes ainsi que l'élasticité de chaque équipe (Travassos, 


\section{eJRIEPS 37 janvier 2016}

Gonçalves, Marcelino, Monteiro, \& Sampaio, 2014).

3. 3. Intérêt et limites

L'intérêt de ces études, pour le chercheur et pour le praticien, est de fournir des données sur les relations entre les contraintes (les variables des SJR: taille, nombre de cible, équilibre numérique, ...) et leurs effets (à un niveau individuel et collectif). De plus, la problématique de la «transférabilité " des acquisitions entre la SJR et la situation de performance (match) a été théorisée à partir du construit de "représentativité », ou de crédibilité SJR. La représentativité, concept initialement proposé par Brunswick (1956), désigne la capacité d'un environnement ou d'un dispositif à produire des comportements crédibles et du transfert. Aussi, la «fidélité » des comportements induits par les SJR par rapport aux comportements observés en situation de performance a été utilisée comme indicateur de la représentativité des SJR (Travassos, Duarte, Vilar, Davids, \& Araujo, 2012). Les résultats montrent que, dans une situation coopérative, des contraintes portant sur le nombre de ballons et sur le nombre de partenaires (nombre d'opportunités de passes offertes) permettraient de se rapprocher du type de passes (précision et vitesse de transmission) produites dans des conditions de match.

Toutefois, cette approche ne peut rendre compte de façon exhaustive de la richesse des SJR pour la formation tactique, dans la mesure où :

le modèle écologique a été utilisé plus comme un modèle de la performance, que comme un modèle de l'apprentissage, même si les chercheurs commencent à s'intéresser à l'évolution du couplage au sein des SJR (Araujo, Davids \& Passos, 2007);

- $\quad$ ces travaux en disent peu sur les mécanismes qui nourrissent le couplage, sur les éléments de l'environnement significatifs qui sous-tendent l'apparition d'invariants parmi les configurations de jeu observées ;

dans les dispositifs de formation, il est difficile de dissocier les moments d'apprentissage implicite et d'apprentissage explicite (Raab, 2007) ; au cours de la pratique réelle des SJR, l'apprenant se nourrit aussi de la co-observation, des échanges verbaux, des conseils entre joueurs ou des feedbacks fournis par le formateur.

\section{Les situations de jeux réduits et le développement de la prise de décision intuitive}

L'utilisation des SJR est plébiscitée au sein de la pédagogie de la compréhension, comme au sein d'une pédagogie par aménagement des contraintes de l'environnement. La présentation des fondements théoriques et l'analyse des effets attendus ont permis de 


\section{eJRIEPS 37 janvier 2016}

pointer un débat relatif à l'usage des SJR. La formation tactique du joueur en sport collectif procède-t-elle d'un apprentissage implicite par adaptation comportementale à l'environnement, ou d'un apprentissage explicite par la compréhension des problèmes posés par ces situations? L'approche théorique de la prise de décision intuitive nous semble pertinente pour prolonger notre analyse dans la mesure où elle valorise à la fois l'apprentissage explicite et l'apprentissage implicite (Raab, 2003, 2007).

4. 1. Fondements théoriques

Dans le cadre du paradigme de la prise de décision intuitive (Klein, 1997, 2008; Raab, 2003, 2007), les chercheurs défendent l'hypothèse d'une diversité de mécanismes développés au cours de l'expérience pour juger et choisir dans des situations complexes. La prise de décision intuitive (PDI) se définit en général en opposition avec la prise de décision rationnelle (PDR) : processus rapide vs lent; holistique vs analytique ; abstrait vs concret (Kahneman \& Klein, 2009). A notre connaissance, deux modèles de la PDI ont été appliqués au sport.

Le modèle de la reconnaissance (Recognition-Primed Decision chez Klein, 1997) décrit la prise de décision comme procédant d'une reconnaissance de configuration spatiotemporelle, fondée sur un ensemble d'éléments contextuels perçus comme pertinents, et spontanément associés à une action typique.

Le modèle de la rationalité écologique (Raab \& Johnson, 2008) est un modèle de la PDI mobilisé par Raab et ses collaborateurs pour étudier les stratégies cognitives permettant d'exploiter la structure de l'information présente dans l'environnement et prendre des décisions acceptables. Ce modèle décrit notamment la prise de décision comme un processus de génération d'options permettant de faire face de façon heuristique aux contraintes variables d'un environnement. En conséquence, Raab (2003) a développé le modèle pédagogique SMART (Situation Model of Anticipated Response consequences of Tactical training) mettant en avant l'intérêt des feedbacks implicites ou explicites pour l'apprentissage, en fonction de la complexité des situations.

4. 2. Processus d'apprentissage et acquisitions

Pour analyser les processus d'apprentissage sollicités par diverses pédagogies de la prise de décision tactique, Raab (2007) utilise la distinction entre apprentissage explicite et implicite. "Quand des règles sont explicitées par l'entraîneur ou si les pratiquants sont incités à expliciter des connaissances " (Raab, id. p.11, notre traduction), on décrit une pratique favorisant un apprentissage explicite. "Quand des règles ou des invariants sont acquis de façon incidente, par la pratique ou l'expérience, sans recours à des 


\section{eJRIEPS 37 janvier 2016}

verbalisations sur les situations » (ibid.), la pratique suscite un apprentissage implicite. Des études en Handball, basket-ball et volley-ball, réalisées en laboratoire, montrent que l'apprentissage implicite conduit à une prise de décision plus pertinente et plus rapide dans des situations peu complexes, et que l'apprentissage explicite permet une prise de décision plus pertinente (mais plus lente) dans des situations complexes (Raab, id.).

Plus généralement, les effets attendus d'une formation tactique à la PDI peuvent être inférés à partir d'études menées sur l'activité d'expert en situation réelle de performance. A partir du modèle de la rationalité écologique les résultats ont montré (a) que les experts en handball choisissent leur première option dans $90 \%$ des situations (Johnson \& Raab, 2003), (b) que le nombre d'options générées est limité à deux ou trois, et que ce nombre est influencé par les émotions (Raab \& Laborde, 2011), (c) enfin que la décision est affectée par les capacités motrices en jeu dans la situation (Raab, 2007).

D'autres travaux ont mobilisé le modèle de la reconnaissance pour analyser la prise de décision chez des experts (e.g., Macquet, 2009). Trois mécanismes de reconnaissance seraient utilisés en fonction des contraintes de l'environnement. En phase offensive 80 à $85 \%$ des situations sont reconnues comme familières, et les joueurs utilisent un mécanisme réactif de reconnaissance simple (simple match), basé sur l'association entre des indices contextuels et la première option de jeu (Bossard, De Keukelaere, Cormier, Pasco, \& Kermarrec, 2010 ; Mulligan, Cracken \& Hodges, 2012). Quand les situations sont perçues comme familières, et que la pression temporelle est relativement faible (e.g., défenseurs éloignés du ballon), les joueurs utilisent des mécanismes de simulation mentale pour vérifier la pertinence de leur option de jeu (dans 3 à $23 \%$ des situations). Quand une situation est perçue comme peu familière ou incertaine, les experts mobilisent un mécanisme de diagnostic pour comparer deux (rarement trois) options de jeu, en mobilisant alors des connaissances (Kermarrec \& Bossard, 2014a).

\section{3. Intérêt et limites}

Le paradigme de la PDI propose une description de la prise de décision en action compatible avec les contraintes de pression temporelle (une ou deux options de jeu envisagées), et prenant en considération la complexité (dimension perceptive, cognitive, motrice, voire émotionnelle) des situations de sports collectifs. Si ces études proposent des résultats empiriques relatifs aux mécanismes de la prise de décision tactiques chez des experts, hormis les travaux de Raab (2003), cette approche a pour le moment rarement été mobilisée dans une perspective de formation en sport (Kermarrec, 2015). Compte tenu des mécanismes identifiés chez les experts, le pédagogue concepteur de 


\section{eJRIEPS 37 janvier 2016}

SJT devrait être attentif à la proximité (familiarité) entre la situation d'apprentissage (SJR) et la situation de performance (jeu réel), et à l'importance des repères spatio-temporels pour faciliter la prise de décision intuitive (Kermarrec \& Bossard, 2014a). Pour Klein (1998) les mécanismes intuitifs se développent au cours de l'expérience à partir de quatre conditions de pratique qui pourraient être réunies dans le cadre des SJR :

une pratique délibérée en fonction d'un but (connaître l'objectif, comprendre l'intérêt de la SJR pour la formation) ;

une compilation intensive d'expériences variées (le jeu induit la motivation nécessaire à la répétition, et une variété de situations) ;

- des feedbacks implicites précis et rapides (fournis dans les SJR par le système de score) ;

une révision de ses expériences et un apprentissage de ses erreurs (le formateur pouvant organiser des pauses dans le jeu pour mettre en évidence une configuration, ou la typicalité d'une situation).

Les propositions de Klein (1998) et les études réalisées par Raab (2003) plaident pour une complémentarité entre l'apprentissage implicite et l'apprentissage explicite. L'usage des SJR pour développer la PDI consisterait en une alternance entre des moments de pratique intensive, et des moments de mise à distance (arrêt du jeu, observation, échanges verbaux), en fonction notamment de la complexité de la SJR. De futures études pourraient chercher à identifier les conditions d'une alternance efficace entre apprentissage explicite et implicite.

Toutefois, ces études relatives à l'expertise en sport collectif ont minoré la dimension collective de l'activité : l'adaptation aux adversaires et la coordination aux partenaires sont nécessaires à la performance et les échanges interindividuels nourrissent l'apprentissage explicite (Darnis \& al., 2006). La recherche en science du sport a mis en évidence les composants cognitifs sous-jacents à la performance collective (e.g. De Keukelaere, Kermarrec, Bossard, Pasco \& De Loor, 2013), composants développés en amont de l'action, connaissances partagées et référentiel commun, ou mobilisés en situation, indices contextuels partagés et mécanismes interactionnels. A notre connaissance, peu d'études ont investigué la construction de coordinations interindividuelles en SJR (e.g., Silva P., Garganta, Araujo, Davids \& Aguiar, 2013). Sur ce point des auteurs suggèrent qu'en valorisant la pratique et l'apprentissage implicite le formateur favoriserait le développement du partage en situation, alors qu'en suscitant des mises à distance ou des confrontations de points de vue entre équipiers, le formateur 


\section{eJRIEPS 37 janvier 2016}

favoriserait le développement de connaissances partagées (Kermarrec \& Bossard, 2014b).

\section{Conclusion}

Le but de cet article était de présenter une synthèse sur l'usage des SJR pour la formation tactique en sports collectifs, à travers trois approches théoriques et pédagogiques. Comme dans toute synthèse interprétative, les conclusions que nous pouvons en tirer ne peuvent constituer une réponse définitive à la question initiale.

5. 1. Trois approches des SJR pour la formation tactique

La pédagogie de la compréhension en sports collectifs valorise la construction par la verbalisation de connaissances spécifiques et de connaissances généralisables, c'est à dire potentiellement réutilisables dans d'autres sports collectifs (Gréhaigne, Richard \& Griffin, 2005). Une des avancées des travaux menés à l'aide du modèle TGfU est de mettre en avant la distinction entre une formation pour la compréhension, que l'on pourrait qualifier d'approche cognitiviste de la formation tactique, et une formation par la compréhension (Gréhaigne \& al., 2010), fondamentalement constructiviste ; cette approche constructiviste mobilise des moments d'apprentissage explicite comme artefacts, et considère les connaissances comme une part de l'arrière-plan décisionnel qui oriente la prise de décision en action (Grehaigne \& Wallian, 2007). De ce point de vue, les SJR offrent des opportunités pour expérimenter des connaissances, connectées avec des habiletés techniques ou motrices: ce sont des situations authentiques, significatives du point de vue de l'apprenant (Kirk \& Mc Phail, 2002).

L'approche de la formation tactique par les contraintes de l'environnement a mis en évidence la stabilisation par la pratique, de relations entre les contraintes des situations et des invariances comportementales individuelles ou collectives. Cette approche fondée sur le modèle écologique défend aussi la spécificité des acquisitions, leur indexation à l'environnement. C'est pourquoi la notion de représentativité des SJR semble particulièrement heuristique (Travassos \& al., 2012) pour viser la transférabilité, la réutilisation des invariants en situation de performance. L'intérêt des travaux est de focaliser l'attention du pédagogue, concepteur de SJR, sur cette représentativité.

Les travaux sur l'expertise menés dans le cadre du paradigme de la prise de décision intuitive (Raab, 2007) mettent en évidence la diversité des mécanismes (processus de reconnaissances et de génération d'options) et des éléments (indices contextuels, connaissances, émotions) sous-tendant les capacités tactiques des joueurs de sports 


\section{eJRIEPS 37 janvier 2016}

collectifs (Kermarrec \& Bossard, 2014a). Des implications pédagogiques ont été proposées (Raab, 2003) qui démontrent les effets différenciés de l'apprentissage implicite et de l'apprentissage explicite. Ces travaux suggèrent que c'est par l'alternance au sein des SJR entre des temps de pratique intensive et des moments d'identification des configurations de jeu significatives, que divers mécanismes de prise de décision tactique pourront être développés.

Les trois approches de la formation tactique présentées ont en commun de s'inscrire en rupture avec l'approche traditionnelle de l'enseignement ou de l'entraînement en sport collectif (Bunker \& Thorpe, 1982 ; Kirk \& Mac Phail, 2002 ; Davids, \& al., 2013). L'approche traditionnelle consiste à solliciter de façon élective les capacités techniques, tactiques ou physiques, et à privilégier la répétition d'un comportement considéré comme efficace lors de situations simples, isolées du contexte de performance. Cette approche caractérisée par Bouthier (1986) comme une pédagogie des modèles d'exécution (PME), renvoie aussi à une « conception techniciste » de l'enseignement des sports collectifs. Cette conception s'avérait dominante chez les praticiens d'après les travaux de BrauAnthony (2001). Au contraire, toutes les approches présentées privilégient l'étude et le développement de l'activité du joueur, appréhendée comme un couplage dynamique et global entre un pratiquant ou ce qu'il a appris (ses habiletés motrices, ses habitudes de jeu, ses connaissances, ses attentes, ses émotions ...) et un ensemble de contraintes (espace de jeu, effectif, temps disponible, cibles, règles et droits ...) qui s'imposent à lui dans l'affrontement présent. La formation tactique reposerait sur le développement de cette capacité de couplage, et plus précisément sur le développement d'une capacité de reconnaissances de configurations de jeu au cours du couplage. Qualifiée de configuration spatio-temporelle dans le modèle RPD (Klein, 2008) ou de configuration prototypique dans l'approche constructiviste de la pédagogie de la compréhension (Gréhaigne \& al., 2014), cette notion de configuration de jeu est au carrefour des trois approches présentées. En outre, dans une perspective de recherche et de formation, elle peut être « objectivée » à partir d'indicateurs ou de repères tels que l'espace de jeu effectif, le circuit préférentiel, l'élasticité du collectif, ou le centroïde de l'équipe (Gréhaigne \& al., 2014 ; Davids \& al., 2013).

Finalement, pour différencier les trois approches théoriques présentées, on peut interroger le rôle de l'intervenant lors de l'usage des SJR. 


\section{eJRIEPS 37 janvier 2016}

5. 2. L'usage des SJR et le rôle de l'intervenant

Nous avons été amenés à interroger les usages des SJR par les intervenants au regard des trois approches théoriques présentées. En effet, même si des rapprochements semblent se dessiner entre ces dernières (Chow et al., 2007 ; Chow et al., 2009), il demeure que les fondements théoriques sous-jacents ne sont pas forcément compatibles. Aussi, les usages des SJR interrogent la façon dont les praticiens mobilisent les théories d'experts (Robin, 1998), ou se réfèrent aux savoirs savants (Terral et Collinet, 2010). Quand un enseignant ou un entraîneur conçoit une SJR, la modifie, régule l'activité du pratiquant, Brau-Anthony montre qu'il mobilise une théorie implicite mêlant des conceptions, croyances, représentations, connaissances technologiques, savoirs professionnels divers. Sensevy (2007) parle d'épistémologie pratique pour souligner que cette théorie d'action peut avoir des conséquences pratiques sur l'usage d'une situation d'apprentissage.

Cette question des fondements théoriques de l'usage des SJR en sports collectifs rappelle par exemple les débats existants à propos des théories de l'apprentissage moteur et de leur utilisation par les intervenants en EPS et en sport (Fargier, 2006). De ce point de vue, la position des intervenants face aux trois approches relatives à la formation tactique en sports collectifs pourrait osciller entre deux possibles: une perspective transpositive et une perspective anthropologique (Fargier, 2006).

Dans une perspective transpositive, nous pourrions imaginer que chaque approche des SJR débouche sur des prescriptions différentes relatives à l'usage des SJR. C'est d'ailleurs le cas quand l'approche TGfU conduit à une structuration des séquences de formation en quatre temps au cours desquels le rôle de l'intervenant est spécifié : faire pratiquer, questionner, aider à verbaliser les règles d'action ou les connaissances construites en action (Gréhaigne \& al., 2001). Dans l'approche écologique, le rôle de l'intervenant se situe essentiellement en amont du couplage, par le design ou la manipulation des contraintes de la situation pour favoriser le couplage perception-action (Davids \& al., 2013). Dans le cadre du paradigme de la prise de décision intuitive, l'alternance entre apprentissage explicite et implicite (Raab, 2007) se traduit par un rôle plus ambigu pour l'intervenant : en amont de l'action, il construit une SJR significative, dont le but ou l'intérêt pour la formation est explicitement identifié ; puis, il incite à l'apprentissage implicite par la pratique intensive, ou interrompt cette pratique pour donner des feedbacks explicites, pour faire partager ou pour renforcer des configurations prototypiques (Kermarrec \& Bossard, 2014b). 


\section{eJRIEPS 37 janvier 2016}

Une seconde perspective qualifiée d'anthropologique conduit à questionner l'usage des SJR en cherchant à dépasser leurs fondements théoriques opposés. Elle s'inspire des travaux de Lévine et Develay (2003) qui considèrent que les intervenants visent la construction de l'expérience humaine en puisant dans sa diversité. Dans cette perspective, l'alternance systématique et volontaire par l'intervenant de différentes pédagogies ou de divers usages des SJR permettrait de confronter les pratiquants à des expériences diversifiées. Dans le cadre de la formation tactique en sport collectif, cette alternance introduit la problématique de l'apprentissage explicite et implicite comme un objet de formation à part entière. Par l'intermédiaire d'une mise à distance de ses expériences (c'est-à-dire les trois formes de pratique des SJR), le pratiquant est amené à s'interroger sur ses propres processus de prise de décision et d'apprentissage (entre apprentissage explicite et implicite). Ce questionnement, qui a par ailleurs une valeur sur le plan de la formation tactique, est intéressant dans cette perspective anthropologique dans la mesure où il permet d'instituer chez le pratiquant une question relative à la part de pilotage «en conscience » de la décision et, plus largement, du comportement humain. Très concrètement, cela conduit à amener un joueur de sport collectif à se questionner sur les moments du jeu où il se laisse «porter par ses intuitions et par l'environnement », et les moments où il s'efforce de « décider de façon lucide ».

\section{3. Perspectives pour la recherche}

La diversité des approches théoriques autour de la question de la formation tactique des joueurs de sports collectifs peut finalement être considérée comme un indicateur de l'intérêt de la question et de sa fécondité pour la recherche en sciences du sport. II semble important d'évaluer et de différencier dans le cadre de l'usage des jeux réduits les effets des contraintes inhérentes aux situations pratiquées, et les effets des aides pédagogiques associées à ces situations (aide à la verbalisation, partage de repères, voir par exemple, Mouchet \& Bouthier, 2006). Par exemple, il serait intéressant d'investiguer les effets de nouvelles technologies (logiciels d'analyse et de modélisation du jeu) actuellement utilisés a priori ou a posteriori (analyse de la performance en compétition ; debriefing), alors qu'ils pourraient constituer des artefacts au cours des jeux réduits pour aider à la reconnaissance des configurations les plus significatives (Kermarrec \& Bossard, 2014b). 


\section{eJRIEPS 37 janvier 2016}

\section{Bibliographie}

Almeida, C.H., Ferreira, A.P., \& Volossovitch, A. (2012). Manipulating Task Constraints in Small-Sided Soccer Games: Performance Analysis and Practical Implications. The Open Sports Sciences Journal, 5, 174-180.

Araújo,D., Davids, K., \& Hristovski, R. (2006). The ecological dynamics of decision making in sport. Psychology of Sport and Exercise, 7 (6), 653-676. doi: 10.1016/j.psychsport.2006.07.002D.,

Araujo, D., Davids, K., \& Serpa, S. (2005). An ecological approach to expertise effects in decision-making in a simulated sailing regatta. Psychology of Sport and Exercise, 6, 671-692.

Araújo, D., Davids, K., \& Passos, P. (2007). Ecological validity, representative design, and correspondence between experimental task constraints and behavioral setting: Ecological Psychology, 19 (1), 69-78. doi: 10.1080/10407410709336951

Araujo, D., Travassos, B., \& Vilar, L. (2010). Tactical skills are not verbal skills : a comment on Kannekens and colleagues. Perceptual and Motor Skills, 110 (3), 1086-1088.

Bossard, C., De Keukelaere, C., Cormier, J., Pasco, D., \& Kermarrec, G. (2010). L'activité décisionnelle en phase de contre-attaque en Hockey sur glace. @ctivités, 7 (1), 41-60.

Bossard, C. \& Kermarrec, G. (2010). La prise de décision des joueurs de sports collectifs : Une revue de question en psychologie du sport. Movement \& Sport Science, 73, 3-22. doi: http://dx.doi.org/10.1051/sm/2010002.

Bouthier, D. (1986). Comparaison expérimentale des effets de différents modèles didactiques des sports collectifs. In EPS, contenus et didactique, (pp. 85-89). Paris : SNEP.

Brau-Antony, S. (2001). Les conceptions des enseignants d'éducation physique et sportive sur l'enseignement et l'évaluation des jeux sportifs collectifs : résultats d'une enquête. Revue STAPS, 56, 93-108.

Brau-Antony, S. \& Hubert, B. (2014). Curriculum en Education Physique et Sportive et évaluation certificative au baccalauréat. Questions Vives, 22, 2-14. DOI : 10.4000/questionsvives.1596

Brunswik, E. (1956). Perception and the representative design of psychological experiments (2nd ed.). Berkeley: University of California Press

Bunker, D., \& Thorpe, R. (1982). A model for teaching of games in secondary schools. 


\section{eJRIEPS 37 janvier 2016}

Bulletin of physical education 19, 5-8.

Butler, J. \& Ovens, A. (2015). TGfU and its governance: from conception to special interest group. Agora for Physical Education and Sport, 17, 1, 77-92.

Chow, J. Y., Davids, K. W., Button, C., Renshaw, I., Shuttleworth, R., Uehara, L. A. (2009) Nonlinear pedagogy : implications for teaching games for understanding (TGfU). In: TGfU : Simply Good Pedagogy : Understanding a Complex Challenge, 14-17 May 2008, University of British Columbia, Vancouver.

Chow, J. Y., Davids, K., Button, C., Shuttleworth, R., Renshaw, I., \& Araújo, D. (2007). The role of nonlinear pedagogy in physical education. Review of Educational Research, 77(3), 251-278.

Collinet, C., Terral, P. (2010). Le développement de la recherche universitaire en EPS depuis 1945 : entre pluralité scientifique et utilité professionnelle. Carrefours de I'Education, 30, 169-186.

Darnis-Paraboschi, F., Lafont, L., \& Menaut, A. (2006). Interactions sociales en dyades symétriques et dissymétriques dans une situation d'apprentissage au handball, Staps, 3 (73), 25-38. DOI : 10.3917/sta.073.38

Davids, K., Araújo, D., Correia, V. \& Vilar, L. (2013). The science of team games: How small-sided games enhance acquisition of movement and decision-making skills. Exercise and Sports Science Reviews, 41 (3), 1-8.

De Keukelaere, C., Kermarrec, G., Bossard, C., G., Pasco, D., \& De Loor, P. (2013). Formes, contenus et évolution du partage au sein d'une équipe sportive de hautniveau. Le Travail Humain, 76. 227-255.

Deleplace, R. (1979). Rugby de mouvement, rugby total. Paris : Revue EPS.

Fargier, P. (2006). EPS et apprentissages moteurs. Paris : Vigot.

Farrow, D., \& Fournier, J. (2005). Training perceptual skill in basketball: Does it benefit the highly skil led? 11th World Congress of International society of Sport Psychology, Sydney, Australia, August 15-19.

Folgado, H., Lemmink, K. A. P. M., Frencken, W. \& Sampaio, J. (2014). Length, width and centroid distance as measures of teams tactical performance in youth football. European Journal of Sport Science, 14,1, 487-492, DOI: $10.1080 / 17461391.2012 .730060$

Gréhaigne, J.F. (1989). Football de mouvement. Vers une approche systémique du jeu. Thèse (non publiée), Université de Bourgogne. 


\section{eJRIEPS 37 janvier 2016}

Gréhaigne, J.F., Billard, M., \& Laroche, J.Y. (1999). L'enseignement des jeux sportifs collectifs à l'école. Conception, construction, évaluation. Bruxelles : De Boeck.

Grehaigne, J., F., Caty, D., \& Godbout, P. (2010). Modelling Ball Circulation in Invasion Team Sports: A Way to Promote Learning Games through Understanding. Physical Education and Sport Pedagogy, 15 (3), 257-270.

Gréhaigne, J.F., Godbout, P., \& Bouthier, D. (2001). The teaching and learning of decision-making in team sports. Quest, 53, 59-76.

Gréhaigne, J.F., Marle, P., \& Zeraï, Z. (2014). Modèles, analyses qualitative et configurations prototypiques dans les sports collectifs. Ejrieps, 30, 5-26.

Gréhaigne, J.F., \& Nadeau, L. (2015). L'enseignement et l'apprentissage de la tactique en sports collectifs : des précurseurs oubliés aux perspectives actuelles. Ejrieps, $35,106-140$.

Gréhaigne, J.F., Richard, J.F., \& Griffin, L. (2005). Teaching and learning team sports and games. New York : RoutledgeFalmer.

Gréhaigne, J.F. \& Roche, J. (1990). Quelques questions à propos du football. In AEEPS (Ed.): Education Physique et Didactique des APS (pp. 64-72).

Gréhaigne, J.F., \& Wallian, N. (2007). Response to Thinck SMART- some elements of perception/decision/action in team sports. Physical Education and Sport Pedagogy, 12 (1), 1-22.

Griffin, L. L., \& Butler, J. I. (2005). Teaching games for understanding. Theory, research and practice. Champaign, IL: Human Kinetics.

Johnson, J.G., \& Raab, M. (2003). Take the first: Option generation and resulting choices. Organizational Behavior and Human Decision Processes, 91, 215-229.

Kahneman D., \& Klein, G. (2009). Conditions for intuitive expertise: A failure to disagree. American Psychologist, 64, 515-526. doi: 10.1037/a0016755

Kermarrec, G. (2015). Enhancing Tactical Skills in Soccer: Advances from the Naturalistic Decision Making Approach. 6th International Conference on Applied Human Factors and Ergonomics, july 2015. Procedia Manufacturing, 3, 1148-1156. http://dx.doi.org/10.1016/j.promfg.2015.07.192

Kermarrec, G., \& Bossard, C. (2014a). Defensive Soccer Players Decision-Making: a Naturalistic Study. Journal of Cognitive Engineering and Decision Making, 8 (2), 187 - 199. doi: 10.1177/1555343414527968.

Kermarrec, G., \& Bossard, C. (2014b). Shared Understanding and Coordination in Team Sports. Contribution of Viewpoints Changes and Shared Information Displays for 


\section{eJRIEPS 37 janvier 2016}

Team Situation Awareness Training. In ICSport 2014-Proceedings of the 2th International Conference on Sport Sciences and Technological Supports, Rome, Italy, October 26-28.

Kirk, D., \& Mc Phail, A. (2002). Teaching Games for understanding and situated learning : rethincking the Bunker-Thorpe model. Journal of Teachning in Physical Education, 21, 177-192.

Klein, G. (1997). The Recognition-Primed Decision (RPD) model: looking back, looking forward. In C.E. Zsambok, \& G.A. Klein (Eds.), Naturalistic Decision Making (pp. 285-292). New Jersey: Lawrence Erlbaum Associates.

Klein, G. (1998). Sources of power: How people make decisions. Cambridge, MA: MIT Press.

Klein, G. (2008). Naturalistic Decision Making, Human Factors, 50 (3), 456-460. doi: $10.1518 / 001872008 \times 288385$

Lévine, J. \& Develay, M. (2003). Pour une anthropologie des savoirs scolaires. Paris : ESF.

Little, T., and Williams, A.G., (2006). Suitability of soccer training drills for endurance training. Journal of Strength and Conditioning Research, 20(2), 316-319

Macquet, A.C. (2009). Recognition within the Decision-Making Process: A Case Study of Expert Volleyball Players. Journal of Applied Sport Psychology, 21(1), 64-80.

Macquet, A.C., \& Fleurance, P. (2006). Des modèles théoriques pour étudier l'activité de l'expert en sport. Science \& Motricité, 58 (2), 9-41.

Masters, R.S.W. (2000). Theoretical aspects of implicit learning in sports. International Journal of Sport Psychology, 31, 530-541.

Merand R. (1989). La rénovation des contenus d'enseignement [Jeux sportifs collectifs au collège]. Revue française de pédagogie, 89, 11-14. doi : 10.3406/rfp.1989.1400

Mitchell, S.A. \& Oslin, J.L. (1999). An investigation of tactical transfer in net games. European Journal of Physical Education, 4, 162-172.

Mouchet, A., \& Bouthier, D. (2006). Prendre en compte la subjectivité des joueurs de rugby pour optimiser l'intervention, Staps, 2, 72, 93-106. DOI 10.3917/sta.072.106

Mouchet A., (2014). Intelligence tactique en sports collectifs. In J.F. Gréhaigne (Ed.), L'intelligence tactique: des perceptions aux décisions tactiques en sports collectifs (pp. 55- 72). Besançon : PUFC. 


\section{eJRIEPS 37 janvier 2016}

Mulligan, D., McCracken, J., \& Hodges, N.J. (2012). Situational familiarity and its relation to decision quality in ice-hockey. International Journal of Sport and Exercise, 10 (3), 198-210. doi: 10.1080/1612197X.2012.672009

Nachon, M. \& Musard, M. (2009) Effet d'une approche constructiviste sur la prise de décisions en éducation physique et sportive : le cas du basket-ball. Science \& Motricité, 1 (66), 25-31. doi : 10.3917/sm.066.0025

Poussin, B., Dénervaud, H. \& Lenzen, B. (2014). La contribution de l'éducation physique au développement de la démarche réflexive des éléves : quelles techniques didactiques mises en œuvre par les enseignants lors d'un cycle de volley-ball ? eJRIEPS, 30, 30-69.

Raab, M. (2003). Decision-making in sports: implicit and explicit learning is affected by complexity of situation. International Journal of Sport and Exercise Psychology, 1, 406-433.

Raab, M., (2007). Thinck SMART, not hard- a review of teaching decision-making in sport from an ecological rationality perspective. Physical Education and Sport Pedagogy, 12 (1), 1-22.

Raab, M., \& Johnson, J. G. (2008). Implicit learning as a means to intuitive decision making in sports. In H. Plessner, C. Betsch, \& T. Betsch (Eds.), Intuition in judgment and decision making (pp. 119-133). Mahwah, NJ: Erlbaum.

Raab, M. \& Laborde, S. (2011): When to Blink and When to Think. Research Quarterly for Exercise and Sport, 82 (1), 89-98, doi :http://dx.doi.org/10.1080/02701367.2011.10599725.

Robin, J-F. (1998). Transposition didactique en gymnastique : les savoirs de référence développés par quatre leaders de théories didactiques. In C. Amade-Escot, J-P. Barrué, J-C Bos, M. Dugrand et A. Terrisse, Recherches en EPS : bilan et perspectives (pp. 109-118). Paris : Editions Revue EPS.

Sensevy, G. (2007). Des catégories pour décrire et comprendre l'action didactique. In G. Sensevy \& A. Mercier (Éds), Agir ensemble. L'action didactique conjointe du professeur et des élèves (pp. 13-49). Rennes : Presses Universitaires de Rennes.

Singleton, E. (2009), From Command to Constructivism: Canadian Secondary School Physical Education Curriculum and Teaching Games for Understanding. Curriculum Inquiry, 39, 321-342. doi: 10.1111/j.1467-873X.2009.00445.x

Silva P., Garganta J., Araujo D., Davids K., \& Aguiar P. (2013). Shared knowledge or 


\section{eJRIEPS 37 janvier 2016}

shared affordances? Insights from an ecological dynamics approach to team coordination in sports. Sports Med, 43, 765-772.

Thorpe, R. (1990). New directions in games teaching. In N. Armstrong (Ed.), New directions in P.E. (Vol. 1, pp. 79-100). Champaign, IL: Human Kinetics.

Travassos, B., Davids, K., Araujo, D., \& Esteves, P. T. (2013). Performance analysis in team sports: Advances from an Ecological Dynamics approach. International Journal of Performance Analysis in Sport, 13 (1), 83-95.

Travassos B, Duarte R, Vilar L, Araujo D., \& Davids K. (2012). Practice task design in team sports: Representativeness enhanced by increasing opportunities for action. Journal of Sports Sciences, 30 (13), 1447-1454.

Travassos, B., Gonçalves, B., Marcelino, R., Monteiro, R. \& Sampaio, J. (2014). How perceiving additional targets modifies teams' tactical behavior during football small-sided games. Human Movement Science, 38, 241-250. Doi : http://dx.doi.org/10.1016/j.humov.2014.10.005

Turner, A. (1996). Myth or reality? Journal of Physical Education, Recreation and Dance, 67(4), 46-49.

Zghibi, M., Guinoubi, C., Bennour, N., \& Moheiddine, N. (2013). Débat d'idées et mise en œuvre des projets d'actions en jeu de Football : cas des garçons de troisième année secondaire en Tunisie. Sport Science Review, 22, 1-2, 151-180. DOI: 10.2478/ssr-2013-0008 